Pacific Journal of Mathematics

FULL CO-ORDINALS OF RETs

ALFRED BERRY MANASTER 


\title{
FULL CO-ORDINALS OF RETS
}

\author{
Alfred B. Manaster
}

\begin{abstract}
Recursive analogues of cardinal and ordinal numbers have been developed by considering only subsets of the natural numbers and considering only one-to-one partial recursive functions as the maps or correspondences between sets. The recursive analogue of a cardinal is called a recursive equivalence type (RET) and that of an ordinal is called a co-ordinal. Using the RETs and the co-ordinals analogues of Cantor's number classes are defined and considered in this paper. The degree of indecomposability of an RET is seen to determine the set of classical ordinals represented in the RET's coordinal number class. If the RET is infinite this set of ordinals is always an initial segment (not necessarily proper) of Cantor's second number class.
\end{abstract}

The basic reference for RETs is Dekker-Myhill [3]. The basic reference for co-ordinals is Crossley [1]. If $\xi$ and $\eta$ are subsets of $E(E=\{0,1,2, \cdots\}), \eta$ is called recursively equivalent to $\xi$ if there exists a one-to-one partial recursive function $f$ whose domain includes $\xi$ such that the $f$-image of $\xi$ is $\eta$. The class of all sets recursively equivalent to $\xi$ is called the recursive equivalence type (RET) of $\xi$ and will be denoted by $\langle\xi\rangle$. If $<_{\xi}$ is a well-ordering of $\xi$ and $<_{\eta}$ is a well-ordering of $\eta$, then $\left(\xi,<_{\xi}\right)$ is called recursively isotonic to $\left(\eta,<_{\eta}\right)$ if there exists a one-to-one partial recursive function $f$ whose domain includes $\xi$ and such that $f$ is an order isomorphism of $\left(\xi,<_{\xi}\right)$ onto $\left(\eta,<_{\eta}\right)$. The class of all-orderings $\left(\eta,<_{\eta}\right)$ recursively isotonic to $\left(\xi,<_{\xi}\right)$ is called the co-ordinal of $\left(\xi,<_{\xi}\right)$ and will be denoted by $\left\langle\xi,<_{\xi}\right\rangle$. If $Y=\left\langle\xi,<_{\xi}\right\rangle$ is a co-ordinal, the classical order type of $\left(\xi,<_{\xi}\right)$ is a countable ordinal which will be referred to as the order type of $Y$ and denoted $|Y|$ (Cf. Definition IV. 2.1 of Crossley [1]).

Addition is defined for RETs and co-ordinals in the following manner. The subsets $\xi_{1}$ and $\xi_{2}$ of $E$ are called RE separable if there exists a pair of disjoint recursively enumerable (RE) sets, $\theta_{1}$ and $\theta_{2}$, such that $\xi_{1} \subseteq \theta_{1}$ and $\xi_{2} \subseteq \theta_{2}$. Assume that $\xi_{1}$ and $\xi_{2}$ are RE separable, $<_{i}$ is a well-ordering of $\xi_{i}$ for $i=1,2$, and $X_{i}=\left\langle\xi_{i}\right\rangle, Y_{i}=\left\langle\xi_{i},<_{i}\right\rangle$ for $i=1,2$. Then $X_{1}+X_{2}=\left\langle\xi_{1} \cup \xi_{2}\right\rangle$ and

$$
Y_{1}+Y_{2}=\left\langle\xi_{1} \cup \xi_{2},<_{1} \cup<_{2} \cup\left(\xi_{1} \times \xi_{2}\right)\right\rangle \text {. }
$$

Using the definitions of addition, partial orderings $\leqq$ have been defined in both the RETs and the co-ordinals. For RETs $X$ and $X_{1}$ define $X_{1} \leqq X$ if and only if there is a RET $X_{2}$ such that $X_{1}+X_{2}=$ $X$. Analogously, for co-ordinals $Y_{1}$ and $Y$ define $Y_{1} \leqq Y$ if and only 
if there is a co-ordinal $Y_{2}$ such that $Y_{1}+Y_{2}=Y$.

Let $Y_{1}=\left\langle\xi_{1},<_{1}\right\rangle$ and $Y_{2}=\left\langle\xi_{2},<_{2}\right\rangle$ be co-ordinals. $Y_{2}$ is called an initial segment of $Y_{1}$ if $\left(\xi_{2},<_{2}\right)$ is recursively isotonic to an initial segment of $\left(\xi_{1},<_{1}\right)$. Definition X. 4.3 of Crossley [2] may now be rephrased as follows. A co-ordinal $Y$ is full if every initial segment of $Y$ is a predecessor of $Y$ in the sense of $\leqq$. Note that if $\left(\xi_{2},<_{2}\right)$ is an initial segment of $\left(\xi_{1},<_{1}\right)$, then $\left\langle\xi_{2},<_{2}\right\rangle \leqq\left\langle\xi_{1},<_{1}\right\rangle$ if and only if $\xi_{2}$ and $\xi_{1}-\xi_{2}$ are RE separable. Thus $\left\langle\xi_{1},<_{1}\right\rangle$ is full if and only if every initial segment of $\left(\xi_{1},<_{1}\right)$ is RE separable from its complement in $\xi_{1}$. Example IV. 5.1 of Crossley [1] shows the existence of coordinals which are not full. The existence of many full co-ordinals is proved in IV. 5.4 of Crossley [1].

There is a natural sense in which the field of a co-ordinal is an RET. To see this consider $\left\langle\xi,<_{\xi}\right\rangle$; if $\left(\xi,<_{\xi}\right)$ is recursively isotonic to $\left(\eta,<_{\eta}\right)$ then $\xi$ is recursively equivalent to $\eta$ and $\langle\xi\rangle=\langle\eta\rangle$, conversely if $\xi$ is recursively equivalent to $\eta$ then there is an ordering $<_{\eta}$ of $\eta$ such that $\left\langle\eta,<_{\eta}\right\rangle=\left\langle\xi,<_{\xi}\right\rangle$. This observation justifies the following definition.

Definition. The field of the co-ordinal $Y=\left\langle\xi,<_{\xi}\right\rangle$ is the RET $X=\langle\xi\rangle$.

In this paper we consider the question of determining the order types of full co-ordinals with a given field $X$. For each RET $X$ let $\mathscr{F}(X)$ be the set of full co-ordinals $Y$ whose field is $X$. Let

$$
\|\mathscr{F}(X)\|=\{|Y|: Y \in \mathscr{F}(X)\}
$$

be the set of order types of full co-ordinals with field $X$.

It will be shown that for each infinite RET $X$ either $\|\mathscr{F}(X)\|=$ $\left[\omega, \omega_{1}\right)$ where $\omega_{1}$ is the first uncountable ordinal or there is a countable positive ordinal $\alpha$ and a finite $n>0$ such that $\|\mathscr{F}(X)\|=\left[\omega, \omega^{\alpha}(n+1)\right)$. $([\alpha, \beta)=\{\gamma: \alpha \leqq \gamma<\beta\} . \quad c$ is the cardinality of the continuum. $)$ For each positive ordinal $\alpha$ and each finite $n>0$ there exist $c$ RETs $X$ such that $\|\mathscr{F}(X)\|=\left[\omega, \omega^{\alpha}(n+1)\right)$. It will also be shown that if the RET $X$ is not an isol (See Chapter IV of Dekker-Myhill [3]) then $\|\mathscr{F}(X)\|=\left[\omega, \omega_{1}\right)$.

A hierarchy structure of the RETs similar to that in Manaster [4] will be useful in demonstrating the results stated above. Note that although the terms and the symbols are similar to those in Definition 0.1 of [4], the definition is slightly different.

Definition. $I_{0}=\{X: X$ is finite $\}$. For each positive countable ordinal $\alpha$ define 


$$
P_{\alpha}=\left\{X: X=Y+Z \Rightarrow Y \in \bigcup_{\beta<\alpha} I_{\beta} \vee Z \in \bigcup_{\beta<\alpha} I_{\beta}\right\}
$$

and

$$
\begin{aligned}
I_{\alpha}= & \left\{X: \text { there is an } n \text { and RETs } X_{1}, \cdots, X_{n}\right. \text { such that } \\
& \text { each } \left.X_{i} \in P_{\alpha} \text { and } X=X_{1}+\cdots+X_{n}\right\} .
\end{aligned}
$$

Elements of $P_{\alpha}$ are called $\alpha$-order indecomposable. Elements of $\bigcup_{\beta<\alpha} I_{\beta}$ are called $\alpha$-small. Elements of $P_{\alpha}$ which are not $\alpha$-small are called strictly $\alpha$-order indecomposable.

In spite of the difference between this definition and definition 0.1 of [4], the two notions of $\alpha$-order indecomposability are similar enough that most of the results of [4] are also correct for this definition of $\alpha$-order indecomposability. If Definition 0.1 of [4] is modified by defining $S_{\alpha}=\{X: X=0\}$, then the two definitions of $P_{\alpha}$ and $I_{\alpha}$ are the same. Replacing some occurrences of $P_{\alpha} \cap S_{\alpha}\left(P_{\alpha}-S_{\alpha}\right)$ with occurrences of $\bigcup_{\beta<\alpha} I_{\beta}\left(P_{\alpha}-\bigcup_{\beta<\alpha} I_{\beta}\right.$ respectively), all results of $\S 1$ of [4] remain valid except Lemma 1.1 and Theorem 1.4. In particular $P_{\alpha}$ is closed under predecessor (Lemma 1.5) so that $I_{\alpha}$ is the ideal generated by $P_{\alpha}$. Moreover the arguments used in Construction I of $\S 2$ and the first part of $\S 3$ (through Theorem 3.2 ) are still valid under the present interpretation and show the existence of $c$ strictly $\alpha$-order indecomposable isols for each countable ordinal $\alpha$.

The main result of this paper is the following theorem.

THEOREM. Let $\alpha$ be a positive countable ordinal. If $X$ is a sum of $n$ strictly $\alpha$-order indecomposables, $\|\mathscr{F}(X)\|=\left[\omega, \omega^{\alpha}(n+1)\right)$. If $X \notin \bigcup_{\alpha<\omega_{1}} I_{\alpha}$, then $\|\mathscr{F}(X)\|=\left[\omega, \omega_{1}\right)$.

Lemma 1. Let $0<\alpha<\omega_{1}$. If $X$ is a sum of $n$-order indecomposables and $Y \in \mathscr{F}(X)$, then $|Y|<\omega^{\alpha}(n+1)$.

Proof. The proof is an induction on $\alpha$. Assume inductively that for $\beta<\alpha$ if $Z$ is a sum of $m \beta$-order indecomposables and $T \in \mathscr{F}(Z)$ then $|T|<\omega^{\beta}(m+1)$. (Note that the remainder of the proof applies for all $\alpha \geqq 1$.) In particular, if $Z$ is $\alpha$-small and $T \in \mathscr{F}(Z)$ then $|T|<\omega^{\alpha}$. Suppose $X=X_{1}+\cdots+X_{n}$ where $n \geqq 1$ and each $X_{i} \in P_{\alpha}$. Suppose $Y \in \mathscr{F}(X)$ and $|Y| \geqq \omega^{\alpha}(n+1)$. Since $Y$ is full, $Y=$ $Y_{1}+\cdots+Y_{n+1}$ where $\left|Y_{i}\right|=\omega^{\alpha}$ for $1 \leqq i \leqq n$ and $\left|Y_{n+1}\right| \geqq \omega^{\alpha}$. Each $Y_{i}$ is full since initial segments and tails of full co-ordinals are full. $X=Z_{1}+\cdots+Z_{n+1}$ where each $Z_{i}$ is the field of $Y_{i}$. By the inductive hypotheses each $Z_{i}$ is not $\alpha$-small since $Y_{i} \in \mathscr{F}\left(Z_{i}\right)$ and $\left|Y_{i}\right| \geqq \omega^{\alpha}$. By the refinement property (Theorem $15(l)$ of Dekker-Myhill [3]) there exist RETs $X_{i, j}$ satisfying the following system of equations. 


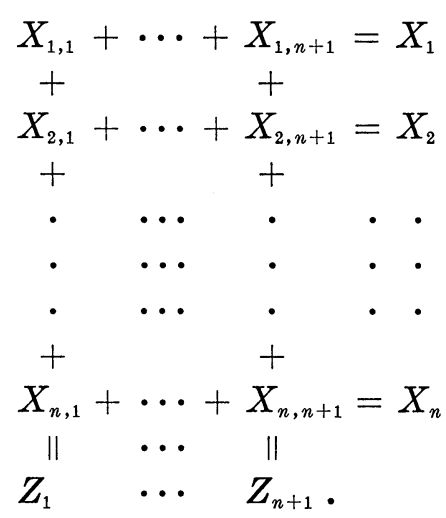

Since each $Z_{j}$ is not $\alpha$-small, for each $j$ there is at least one $i$ such that $X_{i, j}$ is not $\alpha$-small. Since there are $n+1$ columns but only $n$ rows there must be a row, say row $i$, in which there are at least two terms, $X_{i, j}$ and $X_{i, k}$, neither of which is $\alpha$-small. Thus $X_{i} \notin P_{\alpha}$. Contradiction.

Lemma 1 shows that if $X$ is a sum of $n \alpha$-order indecomposables, then $\|\mathscr{F}(X)\| \subseteq\left[\omega, \omega^{\alpha}(n+1)\right)$. The next sequence of lemmas lead to the converse inclusion.

Lemma 2. If $X$ is not $\alpha$-small and $\beta$ is any ordinal less than $\alpha$, then there exist $X_{1}$ and $X_{2}$ such that $X=X_{1}+X_{2}, X_{1}$ is not $\beta$ small, and $X_{2}$ is not $\alpha$-small.

Proof. Suppose $X$ is not $\alpha$-small. Since, in particular, $X \notin P_{\beta}$, there exist $X_{1}$ and $X_{2}$ such that $X=X_{1}+X_{2}$ and neither $X_{1}$ nor $X_{2}$ is $\beta$-small. Not both $X_{1}$ and $X_{2}$ can be $\alpha$-small.

Lemma 3. If $X$ is not $\alpha$-small, then there is a $Y \in \mathscr{F}(X)$ of order type $\omega^{\alpha}$.

Proof. The proof is an induction on $\alpha$. The base step, $\alpha=1$, is easy since every infinite RET is the field of full co-ordinals of type $\omega$. Let $1<\alpha<\omega_{1}$. Let $\left\{\beta_{i}\right\}_{i<\omega}$ be a sequence of ordinals such that $1 \leqq \beta_{0} \leqq \beta_{1} \leqq \beta_{2} \leqq \cdots<\alpha$ and such that for every $\beta<\alpha$ there is an $i$ such that $\beta \leqq \beta_{i}$. (If $\alpha=\gamma+1$, let each $\beta_{i}=\gamma_{\text {. }}$ )

Since $X$ is not $\alpha$-small and $\beta_{0}<\alpha$, by Lemma 2 there exist $X_{0}$ and $Z_{0}$ such that $X=X_{0}+Z_{0}, X_{0}$ is not $\beta$-small, and $Z_{0}$ is not $\alpha$-small. Inductively for each $n, X=X_{0}+\cdots+X_{n}+Z_{n}$ where $Z_{n}$ is not $\alpha$-small. Since $\beta_{n+1}<\alpha$ by Lemma 2 there exist $X_{n+1}$ and $Z_{n+1}$ such that $Z_{n}=X_{n+1}+Z_{n+1}, X_{n+1}$ is not $\beta_{n+1}$-small and $Z_{n+1}$ is not $\alpha$-small. Thus

$$
X=X_{0}+\cdots+X_{n}+X_{n+1}+Z_{n+1}
$$


where each $X_{i}$ is not $\beta_{i}$-small and $Z_{n+1}$ is not $\alpha$-small.

Since $\beta_{n}<\alpha$ the inductive hypothesis asserts the existence of a full co-ordinal $Y_{n}$ of order type $\omega^{\beta}$ with field $X_{n}$. Unfortunately the ordinal sum of the $Y_{n}$ is not well defined and even if it were it would not, in general, be a co-ordinal with field $X$. However, it would be a full co-ordinal of type $\omega^{\alpha}$. To remove these difficulties, it seems necessary to work with a representative of $X$.

Let $\langle\xi\rangle=X$. For each $n$ let $\xi_{n}$ be a representative of $X$ such that $\bigcup_{n} \xi_{n} \subseteq \xi$ and $\bigcup_{i=1}^{n} \xi_{i}$ is $\mathrm{RE}$ separable from $\xi-\bigcup_{i=1}^{n} \xi_{i}$. Define

$$
\xi_{n}^{\prime}=\xi_{n} \cup\left(\{n\} \cap \xi \cap \overline{\bigcup_{m<n} \xi_{m}}\right)
$$

and $X_{n}^{\prime}=\left\langle\xi_{n}^{\prime}\right\rangle$. For any RET $Z$ both $Z$ and $Z+1$ have the same order of indecomposability, if any. Thus $X_{n}^{\prime}$ is not $\beta_{n}$-small. Now apply the inductive hypothesis to obtain full co-ordinals $Y_{n}^{\prime}$ of order type $\omega^{\beta}$ with field $X_{n}^{\prime}$. Let $Y_{n}^{\prime}=\left\langle\xi_{n}^{\prime},<_{n}\right\rangle$ and define

$$
Y=\left\langle\bigcup_{n<\omega} \xi_{n}^{\prime}, \bigcup_{n<\omega}<_{n} \cup \bigcup_{m<n}\left(\xi_{m}^{\prime} \times \xi_{n}^{\prime}\right)\right\rangle \text {. }
$$

$Y$ is a full co-ordinal of type $\sum \omega^{\beta_{n}}=\omega^{\alpha}$ with field $X$.

Lemma 4. If $Y \in \mathscr{F}(X),|Y|=\alpha$, and $\omega \leqq \beta<\alpha$, then there is a $Y^{\prime} \in \mathscr{F}(X)$ such that $\left|Y^{\prime}\right|=\beta$.

Proof. First consider the case in which $\alpha-\beta$ is finite. $Y=$ $Y_{1}+Y_{2}$ where $\left|Y_{1}\right|=\beta$ and $\left|Y_{2}\right|=\alpha-\beta<\omega . \quad Y^{\prime}=Y_{2}+Y_{1}$ is then a full co-ordinal of type $(\alpha-\beta)+\beta=\beta$ with field $X$.

Next consider the case in which $\alpha-\beta \geqq \omega$ and $\omega^{2} \leqq \beta . \quad Y=$ $Y_{1}+Y_{2}$ where $\left|Y_{1}\right|=\beta$ and $\left|Y_{2}\right|=\alpha-\beta \geqq \omega$. Let $Y_{2}^{\prime}$ be a (necessarily full) ordering of type $\omega$ of the field of $Y_{2}$. $\quad Y^{\prime}=Y_{2}^{\prime}+Y_{1}$ satisfies the conclution of the lemma.

Finally consider the case in which $\alpha-\beta \geqq \omega$ and $\omega^{2}>\beta$. Observe that for every $k<\omega$, any co-ordinal of type $\omega+k$ is full. Let $\beta=\omega n+k$ where $n$ and $k$ are finite. If $n=1$ the field of $Y$ has full co-ordinals of type $\beta$ by the observation just made. Otherwise let $n=m+1$ where $m \geqq 1 . \quad Y=Y_{1}+Y_{2}$ where $\left|Y_{1}\right|=\omega m$ and

$$
\left|Y_{2}\right|=\alpha-\omega m \geqq \alpha-(\omega n+k)=\alpha-\beta \geqq \omega .
$$

Let $Y_{2}^{\prime}$ be a full co-ordinal of type $\omega+k$ with the same field as the field of $Y_{2} . \quad Y=Y_{1}+Y_{2}^{\prime}$ is a full co-ordinal of type $\omega m+\omega+k=\beta$ with field $X$.

Lemma 5. If $X$ is not $\alpha$-small then $\left[\omega, \omega^{\alpha}\right] \subseteq\|\mathscr{F}(X)\|$. 


\section{Proof. Apply Lemmas 3 and 4.}

Proof of theorem. First suppose $X$ is not $\alpha$-small for any countable ordinal $\alpha$. $\omega^{\alpha} \in\|\mathscr{F}(X)\|$ for each countable $\alpha$ by Lemma 3 . Thus $\|\mathscr{F}(X)\|=\left[\omega, \omega_{1}\right)$ by Lemma 4 .

To prove that if $X$ is a sum of $n$ strictly $\alpha$-order indecomposables than $\|\mathscr{F}(X)\|=\left[\omega, \omega^{\alpha}(n+1)\right)$, consider first the case $n=1$. Since $X$ is not $\alpha$-small there is, by Lemma 3, a full co-ordinal $Y$ with field $X$ of order type $\omega^{\alpha}$. Let $\omega^{\alpha} \leqq \beta<\omega^{\alpha} \cdot 2$. For some $\gamma<\omega^{\alpha}, \beta=\omega^{\alpha}+\gamma$. Let $Y=Y_{1}+Y_{2}$ where $\left|Y_{1}\right|=\gamma$ and $\left|Y_{2}\right|=\omega^{\alpha}-\gamma=\omega^{\alpha} . \quad Y^{\prime}=$ $Y_{2}+Y_{1}$ is a full co-ordinal with field $X$ of type $\left|Y_{2}\right|+\left|Y_{1}\right|=$ $\omega^{\alpha}+\gamma=\beta$. This result, Lemma 5 , and Lemma 1 show $\|\mathscr{F}(X)\|=$ $\left[\omega, \omega^{\alpha} \cdot 2\right)$.

Finally consider the case in which $X=X_{1}+\cdots+X_{n}, n \geqq 2$, and each $X_{i}$ is strictly $\alpha$-order indecomposable. Let

$$
\beta \in\left[\omega^{\alpha} \cdot n, \omega^{\alpha}(n+1)\right) .
$$

For some $\gamma<\omega^{\alpha}, \beta=\omega^{\alpha} \cdot n+\gamma$. Let $Y_{i}$ be a full co-ordinal with field $X_{i}$ of order type $\omega^{\alpha}$ for each $i<n$. Let $Y_{n}$ be a full co-ordinal of order type $\omega^{\alpha}+\gamma$ with field $X_{n} . \quad Y=Y_{1}+\cdots+Y_{n}$ is a full co-ordinal of type $\beta$ with field $X$. This result, Lemma 4, and Lemma 1 show $\|\mathscr{F}(X)\|=\left[\omega, \omega^{\alpha}(n+1)\right)$.

It remains to show that if the RET $X$ is not an isol then $\|\mathscr{F}(X)\|=$ $\left[\omega, \omega_{1}\right)$ and show the existence of $c$ isols such that $\|\mathscr{F}(X)\|=$ $\left[\omega, \omega_{1}\right)$. For the former result it suffices to show that if $X$ is not an isol then $X \notin \bigcup_{\alpha<\omega_{1}} I_{\alpha}$ and hence it suffices to show that $X \notin \bigcup_{\alpha<\omega_{1}} P_{\alpha}$. Let $\alpha$ be the least ordinal $\beta$ such that some non-isol $X$ is $\beta$-order indecomposable. $X=X_{1}+X_{2}$ where neither $X_{1}$ nor $X_{2}$ is an isol but one of them, say $X_{1}$, is a finite sum of $\beta$-order indecomposables for some $\beta<\alpha$. Since $\beta<\alpha$ every $\beta$-order indecomposable is an isol. Since every finite sum of isols is an isol, $X_{1}$ must be an isol. This contradiction shows that every nonisol is not in $\bigcup_{\alpha<\omega_{1}} I_{\alpha}$.

Examples of isols $X$ such that $\|\mathscr{F}(X)\|=\left[\omega, \omega_{1}\right)$ are provided by first-order highly decomposable isols in the sense of Manaster [4]. It will be shown, as in the preceding paragraph, that if $X$ is firstorder highly decomposable then $X \notin \bigcup_{\alpha<\omega_{1}} P_{\alpha}$. Let $\alpha$ be the least ordinal $\beta$ such that some first-order highly decomposable $X$ is $\beta$-order indecomposable. $X=X_{1}+X_{2}$ where both $X_{1}$ and $X_{2}$ are infinite but one, say $X_{1}$, is a finite sum of $\beta$-order indecomposables for some $\beta<\alpha$. Since $X_{1}$ is infinite, there is an infinite $X_{3} \leqq X_{1}$ such that $X_{3}$ is $\beta$ order indecomposable. Since $X_{3} \leqq X_{1} \leqq X, X_{3}$ is highly decomposable contradicting the minimality of $\alpha$. The existence of $c$ first-order highly decomposable isols is shown in Dekker-Myhill [3, pp. 112-113] 
and Manaster [4]. Thus there are $c$ isols $X$ such that $\|\mathscr{F}(X)\|=$ $\left[\omega, \omega_{1}\right)$.

\section{REFERENCES}

1. J. N. Crossley, Constructive order types, I, in Formal Systems and Recursive Functions, Proc. of the Eighth Logic Colloquium, Oxford 1963, Ed. by J. N. Crossley and M. A. E. Dummett, Amsterdam (1965), 189-264.

2. Constructive order types, II, Journal of Symbolic Logic 31 (1966), 525-538. 3. J. C. E. Dekker and J. Myhill, Recursive equivalence types, University of California Publications in Mathematics (New Series) 3 (1960), 67-214.

4. A. B. Manaster, Higher-order indecomposable isols, Trans. Amer. Math. Soc. 125 (1966), 363-383.

Received May 23, 1967.

Massachusetts Institute of Technology 



\section{PACIFIC JOURNAL OF MATHEMATICS}

\section{EDITORS}

\section{H. ROYDEN}

Stanford University

Stanford, California

R. R. Phelps

University of Washington

Seattle, Washington 98105

\section{J. DugundJI}

Department of Mathematics

University of Southern California

Los Angeles, California 90007

RICHARD ARENS

University of California

Los Angeles, California 90024

\section{ASSOCIATE EDITORS}
E. F. BeCKENBACH
B. H. NeumanN
F. WOLF
K. YosIDA

\section{SUPPORTING INSTITUTIONS}

\author{
UNIVERSITY OF BRITISH COLUMBIA \\ CALIFORNIA INSTITUTE OF TECHNOLOGY \\ UNIVERSITY OF CALIFORNIA \\ MONTANA STATE UNIVERSITY \\ UNIVERSITY OF NEVADA \\ NEW MEXICO STATE UNIVERSITY \\ OREGON STATE UNIVERSITY \\ UNIVERSITY OF OREGON \\ OSAKA UNIVERSITY \\ UNIVERSITY OF SOUTHERN CALIFORNIA
}

\author{
STANFORD UNIVERSITY \\ UNIVERSITY OF TOKYO \\ UNIVERSITY OF UTAH \\ WASHINGTON STATE UNIVERSITY \\ UNIVERSITY OF WASHINGTON \\ AMERICAN MATHEMATICAL SOCIETY \\ CHEVRON RESEARCH CORPORATION \\ TRW SYSTEMS \\ NAVAL WEAPONS CENTER
}

Mathematical papers intended for publication in the Pacific Journal of Mathematics should be in typed form or offset-reproduced, double spaced with large margins. Underline Greek letters in red, German in green, and script in blue. The first paragraph or two must be capable of being used separately as a synopsis of the entire paper. It should not contain references to the bibliography. Manuscripts, in duplicate if possible, may be sent to any one of the four editors. All other communications to the editors should be addressed to the managing editor, Richard Arens, University of California, Los Angeles, California 90024.

Each author of each article receives 50 reprints free of charge; additional copies may be obtained at cost in multiples of 50 .

The Pacific Journal of Mathematics is published monthly. Effective with Volume 16 the price per volume (3 numbers) is $\$ 8.00$; single issues, $\$ 3.00$. Special price for current issues to individual faculty members of supporting institutions and to individual members of the American Mathematical Society: $\$ 4.00$ per volume; single issues $\$ 1.50$. Back numbers are available.

Subscriptions, orders for back numbers, and changes of address should be sent to Pacific Journal of Mathematics, 103 Highland Boulevard, Berkeley 8, California.

Printed at Kokusai Bunken Insatsusha (International Academic Printing Co., Ltd.), 7-17, Fujimi 2-chome, Chiyoda-ku, Tokyo, Japan.

PUBLISHED BY PACIFIC JOURNAL OF MATHEMATICS, A NON-PROFIT CORPORATION

The Supporting Institutions listed above contribute to the cost of publication of this Journal, but they are not owners of publishers and have no responsibility for its content or policies. 


\section{Pacific Journal of Mathematics}

\section{Vol. 26, No. $3 \quad$ BadMonth, 1968}

Leonard Asimow, Universally well-capped cones ................. 421

Lawrence Peter Belluce, William A. Kirk and Eugene Francis Steiner,

Normal structure in Banach spaces ..................... 433

William Jay Davis, Bases in Hilbert space.................... 441

Larry Lee Dornhoff, p-automorphic p-groups and homogeneous

algebras..................................... 447

William Grady Dotson, Jr. and W. R. Mann, A generalized corollary of the

Browder-Kirk fixed point theorem ....................... 455

John Brady Garnett, On a theorem of Mergelyan ................. 461

Matthew Gould, Multiplicity type and subalgebra structure in universal

algebras............................................. 469

Marvin D. Green, A locally convex topology on a preordered space . . . . . 487

Pierre A. Grillet and Mario Petrich, Ideal extensions of semigroups . . . . . . 493

Kyong Taik Hahn, A remark on integral functions of several complex

variables ... . . . . . . . . . . . . . . . . . . . . . . . . . . . . . . . . . . . 509

Choo Whan Kim, Uniform approximation of doubly stochastic

operators..................................... 515

Charles Alan McCarthy and L. Tzafriri, Projections in $\mathscr{L}_{1}$ and

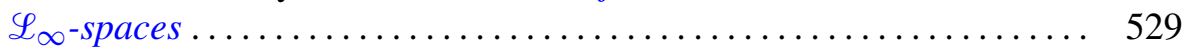

Alfred Berry Manaster, Full co-ordinals of RETs ................ 547

Donald Steven Passman, $p$-solvable doubly transitive permutation

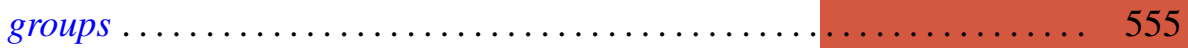

Neal Jules Rothman, An $L^{1}$ algebra for linearly quasi-ordered compact

semigroups ....................................... 579

James DeWitt Stein, Homomorphisms of semi-simple algebras .......... 589

Jacques Tits and Lucien Waelbroeck, The integration of a Lie algebra

representation ...............................

David Vere-Jones, Ergodic properties of nonnegative matrices. II ........ 601

Donald Rayl Wilken, The support of representing measures for $R(X) \ldots \ldots 621$

Abraham Zaks, Simple modules and hereditary rings .... . . 